\title{
Analysis of Operating Conditions Influencing the Morphology and In vitro Behaviour of Chitosan Coated Liposomes
}

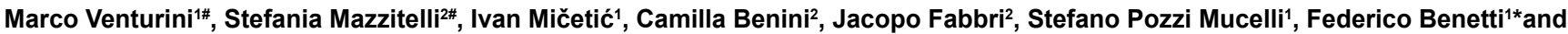 \\ Claudio Nastruzzi ${ }^{2 *}$
}

${ }^{1}$ ECSIN-European Center for the Sustainable Impact of Nanotechnology, Veneto Nanotech S.C.p.A., Viale Porta Adige 45, I-45100 Rovigo, Italy

${ }^{2}$ Department of Life Sciences and Biotechnology, University of Ferrara, Via Fossato Mortara 17/19, Ferrara 44121, Italy

\#These authors contributed equally to the study

\begin{abstract}
Among nanoparticle-based drug delivery formulations, lecithin/chitosan liposomes are promising candidates because of their biocompatibility, biodegradability and bioadhesion properties. Lecithin is a mixture of highly biocompatible phospholipids, while chitosan represents one of the most used polymers in pharmaceutical formulations. Their combination results in positively charged complexes that are able to sustain a specific, prolonged and controlled release. Scarce reproducibility and batch-to-batch variation in lecithin/chitosan liposome synthesis, as well as difficult scale-up to industrial production, is a major challenge for their utilization. Here we present a strictly controlled procedure, based on ethanol diffusion in water, which yields to a precise and reproducible self-organization of lecithin and chitosan molecules. We analysed the size, surface charge and stability of chitosan coated liposomes at different lecithin/ chitosan ratios. We found that increasing the lecithin/chitosan ratio both mean particle size and surface charge were progressively reduced. A good stability was observed for all formulations, though interactions occurred in liposomes with low amount of surface-adsorbed chitosan. Chitosan coated liposomes interact with A549 and Caco-2 cells inducing low toxic effects only with prolonged incubation times. In conclusion, the proposed procedure provides good reproducibility in the formation of non-toxic and highly stable formulations of chitosan coated liposomes as drug delivery systems.
\end{abstract}

Keywords: Lecithin; Chitosan; Liposomes; Ethanol injection procedure; Dynamic light scattering; Transmission electron microscopy; Cytotoxicity

\section{Introduction}

Delivering therapeutic compounds to target sites is a major challenge in treatment of many diseases [1,2]. Nanosized materials in form of nanoparticles, micelles and liposomes are among the most promising drug delivery systems for the transport of pharmaceuticals to the site of action, reducing toxicity and side effects associated to several drugs $[3,4]$. In particular, nanocarriers with appropriate physicochemical characteristics such as dimensions and surface charge have been recently investigated as delivery systems able to protect drugs from enzymatic degradation, improve drug pharmacokinetics and enter cells and various cellular compartments [5-7].

Nanoparticles can be constituted of lipids, degradable or nondegradable polymers, metals and organic/inorganic compounds [8-10]. Among these classes, special attention has been drawn to lipids such as lecithin and degradable polymers such as chitosan for their outstanding physical and biological properties [11,12]. Lecithin is a natural lipid mixture of phospholipids resulting in a highly biocompatible material, widely used for preparation of various nanoparticulate delivery systems including liposomes $[13,14]$. Chitosan, poly( $\beta$ - $(1,4)-2$-amino-2deoxy-D-glucose), is one of major polymers used in pharmaceutical formulations [15]. Chitosan is obtained by alkaline deacetylation of chitin, which exists in the natural structure of crustacean exoskeletons [16]. For its multiple properties such as biocompatibility, biodegradability, bioadhesion and antimicrobial activity, chitosan has been widely employed in pharmaceutical, food and cosmetics fields $[17,18]$. Several studies have highlighted the potential of chitosan as an absorption-enhancing agent. It has also received considerable attention in novel bioadhesive drug delivery systems with the aim of prolonging residence time at the administration site $[19,20]$. Chitosan has also the potential to electrostatically interact with negatively charged lipids such as lecithin, spontaneously forming supramolecular aggregates [21]. The appropriate combination of lecithin with chitosan results in positively charged complexes with specific, prolonged and controlled release characteristics. Several authors have described the use of chitosan as a coating material for liposomes, resulting in formulation with an improved oral bioavailability for peptide and protein drugs [22-25]. Chitosan-coated liposomes (CCL) containing insulin have been shown to produce hypoglycaemic effects in mice after oral administration [26]. Moreover, lecithin-chitosan nanoparticles can strongly interact with mammalian skin, which is slightly negatively charged, offering a better topical delivery of active compounds $[27,28]$. On the contrary, in conventional uncoated liposomes without any surface modification, the lack of positive charges does not ensure close contact between nanoparticles and skin. As a consequence, uncoated liposomes are rapidly removed from skin surface.

These findings suggest that CCL possess a great potential as drug delivery systems improving the permeation of entrapped drugs across various biological barriers including skin and mucosal tissue [23,29$31]$.

*Corresponding author: Claudio Nastruzzi, Department of Life Sciences and Biotechnology, University of Ferrara, Via Fossato di Mortara 17/19, 44100, Ferrara, Italy, Tel: (+39) 0532 455348; E-mail: nas@unife.it

Federico Benetti, ECSIN-European Center for the Sustainable Impact of Nanotechnology, Veneto Nanotech S.C.p.A., Viale Porta Adige 45, I-45100 Rovigo, Italy, Tel: (+39) 0425 377512; E-mail: federico.benetti@venetonanotech.it

Received June 04, 2014; Accepted July 17, 2014; Published July 21, 2014

Citation: Venturini M, Mazzitelli S, Mičetić I, Benini C, Fabbri J, et al. (2014) Analysis of Operating Conditions Influencing the Morphology and In vitro Behaviour of Chitosan Coated Liposomes. J Nanomed Nanotechnol 5: 211. doi: 10.4172/2157-7439.1000211

Copyright: @ 2014 Venturini M, et al. This is an open-access article distributed under the terms of the Creative Commons Attribution License, which permits unrestricted use, distribution, and reproduction in any medium, provided the original author and source are credited. 
The current work reports the production of chitosan coated liposomes (CCL) by a controlled ethanol injection of lecithin into a chitosan/water solution. Different papers have indeed described the preparation of CCL, but none was based on a strict control of experimental parameters. Injection of lecithin ethanolic solution into the chitosan solution is performed in various uncontrolled methods, mainly by hand pipetting through a glass pipette. In addition, during the injection, the stirring is generally accomplished by unstandardized procedure based on magnetic stirrer, without defining the magnetic bar and reactor dimensions. Therefore, preparation techniques currently described in literature appear to be scarcely reproducible and not suitable for large scale manufacturing, due to batch-to-batch variations.

The present study focuses on analysis of strict control operating conditions influencing the morphology and in vitro behaviour of chitosan coated liposomes. CCL were prepared by injecting ethanol solution with highly purified phosphatidylcholine enriched lecithin (min. 85.0\%) into a water phase by a syringe pump in a controlled fashion. The obtained nanoparticles were characterised in terms of size, zeta potential and morphology. Finally, due to possible applications of CCL as carriers for drug delivery, we also performed a toxicological evaluation on A549 and Caco-2 cells, as in vitro models of human lung and intestinal epithelia.

\section{Materials and Methods}

\section{Materials}

Reagents used for the nanoparticle preparations were: phosphatidylcholine from soybean (85\%) (Phospholipon 85G, Lipoid $\mathrm{GmbH}$, Germany), chitosan from shrimp shells ( $\geq 85 \%$ deacetylated, viscosity $100 \mathrm{cP}, 1 \% \mathrm{w} / \mathrm{v}$ solution in $1 \% \mathrm{v} / \mathrm{v}$ acetic acid) (SigmaAldrich, UK) and 5(6)-Carboxyfluorescein (Sigma-Aldrich, UK). All other regents were from Sigma-Aldrich, UK and were of analytical grade. Reagents used for cytotoxicity assays were: CellTiter 96 AQueous Non-Radioactive Cell Proliferation Assay kit (Promega, Madison, WI, USA), cytotoxicity detection kit plus (Roche, Mannheim Germany) and ATPlite (Perkin Elmer, Waltham, MA, USA). Human lung carcinoma epithelial cells A549 (\#CCL-185) and human colorectal adenocarcinoma cells Caco-2 (HTB-37) were purchased from American Type Culture Collection (Manassas, VA, USA). A549 cells were cultured in Ham's F12 medium, fetal bovine serum (FBS), penicillin-streptomycin solution, L-glutamine, phosphate buffered saline (PBS), Dulbecco's phosphate buffered saline with calcium and magnesium (DPBS), all purchased from Lonza (Basel, Switzerland). Caco-2 cells were cultured in Dulbecco's modified eagle medium (DMEM) purchased from Life Technologies (Carlsbad, CA). Sodium dodecyl sulfate (SDS), staurosporine (STS) and glycerol were purchased from Sigma-Aldrich (St. Louis, MO, USA).

\section{Chitosan coated liposome preparation}

Lecithin $(30 \mathrm{mM})$ was solubilized in $4 \mathrm{~mL}$ of ethanol and injected into $36 \mathrm{~mL}$ of $1 \%$ acetic acid solution containing different amounts of chitosan, namely: $0.456,0.228,0.114,0.045,0.033$ or $0.022 \mathrm{mg} / \mathrm{mL}$, resulting in a final lecithin/chitosan ratio of 5, 10, 20, 50, 75 and 100, respectively. Ethanol injection was accomplished by a $10 \mathrm{~mL}$ syringe connected with a PTFE tube (internal diameter $0.7 \mathrm{~mm}$ ) and a syringe pump at constant flow-rate of $1 \mathrm{~mL} / \mathrm{min}$. The organic solution was injected into the chitosan water phase placed into a glass reactor $(50 \times 90$ $\mathrm{mm}$, diameter/height), using a mechanical stirrer (model IKA RW 20 DZM) equipped with 3-bladed impeller (40 $\mathrm{mm}$ diameter). The same procedure was employed for the preparation of carboxyfluorescein
(CF) loaded CCL where a $0.1 \%$ CF (w/v) solution was added to initial $4 \mathrm{~mL}$ of lecithin.

\section{Determination of CF loading into CCL}

Free CF was separated from CF loaded CCL by size exclusion chromatography using a Sepharose 4B column (Pharmacia, Uppsala, Sweden) $(1.5 \mathrm{~cm}$ diameter, $50 \mathrm{~cm}$ length) equilibrated with borate buffer. The void volume peak fractions containing CF loaded CCL were collected and quantified for liposome and free CF content. UVVIS spectrum of each fraction was measured both in isotonic Palitzsch buffer and in ethanol/water (70:30) mixture in order to estimate both the suspension turbidity (from optical density at $600 \mathrm{~nm}$, when measured in buffer), and determine the concentration of CF (from optical density at $310 \mathrm{~nm}$ of optically transparent ethanol/water). All UV spectra were recorded on a Hewlett-Packard 8452 diode array spectrophotometer. Total and free amounts of resulting $\mathrm{CF}$ (C0 and $\mathrm{C} 1$, respectively) were used to calculate the encapsulation efficiency (EE) as follows:

$$
\mathrm{EE}=(1-\mathrm{C} 1 / \mathrm{C} 0) \times 100 .
$$

\section{CCL characterisation}

Dynamic laser light scattering: Particle sizes of nanoparticle formulations were measured by dynamic laser light scattering method with Zetasizer 3000 PCS instrument (Malvern Instruments, UK). The analysis was performed using a 5-mW helium neon laser with a wavelength output of $633 \mathrm{~nm}$. Glassware was cleaned of dust by washing with detergent and rinsing twice with water for injections. Measurements were made at $25^{\circ} \mathrm{C}$, at an angle of $90^{\circ}$. Data were elaborated using the Contin software.

Particle size distributions of CCL suspensions were also measured with Malvern ZetasizerNano ZS DLS instrument operated in backscattering mode. Medium viscosity of $0.89 \mathrm{cP}$ and refractive indices of 1.590 (CCL) and 1.330 (water) were assumed. Measurements were carried at $23^{\circ} \mathrm{C}$ and were run in triplicate to check for sedimentation and solution stability.

Transmission electron microscopy: CCL morphology was investigated by negative-stain transmission electron microscopy (TEM) with a FEI Tecnai 12 G2 electron microscope with Twin lens configuration (FEI Co., Eindhoven, The Netherlands). CCL suspension was diluted 100 -fold with water and mixed with equal volume of a $2 \%$ (w/v) ammonium molybdate solution. Formvar-coated copper grids were placed on a drop of sample solutions and were left to incubate for 5 minutes. Excess of CCL suspensions was blotted from grids using filter paper, leaving only a thin film layer on a small part of a grid. CCL samples were observed following complete evaporation of solvents from the grid. Samples were imaged at $120 \mathrm{kV}$ and micrographs were recorded with a side-mounted Olympus Morada CCD (Olympus Soft Imaging Solutions GmbH, Münster, Germany).

\section{CCL zeta potential determination}

The formation of chitosan layer on liposomal surface was analysed by zeta potential measurements of vesicle surface. Zeta potential of CCL was determined using a Zetasizer Nano ZS (Malvern Instruments, UK). Measurements were run in triplicate for each type of CCL.

\section{CCL stability}

Stability of CCL preparations at different lecithin/chitosan ratios was evaluated at $25^{\circ} \mathrm{C}$ over time. Samples were sealed in glass bottles containing a water/ethanol $(90: 10)$ solution and stored at $25^{\circ} \mathrm{C}$ for a 
period of 60 days. Periodically sample aliquots were collected for particle size analysis by DLS.

\section{In vitro cell cultures}

Human lung carcinoma epithelial A549 cells, used from passages 88-105, were maintained in Ham's F12, 10 \% FBS, 2mM L-glutamine, $100 \mu \mathrm{g} / \mathrm{mL}$ penicillin, $100 \mathrm{U} / \mathrm{mL}$ streptomycin. In vitro cytotoxicity assays were performed in 96-well microplates seeding $5 \times 10^{3}$ cells/well. Human colorectal adenocarcinoma Caco-2 cells, used from passages 35-45, were maintained in DMEM high glucose, $10 \%$ heat inactivated FBS $2 \mathrm{mM}$ L-glutamine, $100 \mu \mathrm{g} / \mathrm{mL}$ penicillin, $100 \mathrm{U} / \mathrm{mL}$ streptomycin. In vitro assays were performed in 96-well microplates seeding $1 \times 10^{4}$ cells/well. Fluorescence microscopy was performed on A549 and Caco- 2 cells seeded on glass coverslips, in 6-well plates for suspension cultures, at $1 \times 10^{5}$ cells/well and $3 \times 10^{5}$ cells/well respectively. Both cell lines were grown in a $5 \% \mathrm{CO}_{2}$ incubator in humidified atmosphere at $37^{\circ} \mathrm{C}$.

\section{Cytotoxicity tests}

Cellular viability was determined by measuring mitochondrial activity and adenosine triphosphate content (ATP), while membrane integrity was assessed quantifying lactate dehydrogenase (LDH) released into medium from damaged cells. Briefly, $24 \mathrm{~h}$ after seeding cells were exposed to increasing CCL concentrations for $2 \mathrm{~h}, 6 \mathrm{~h}$, $24 \mathrm{~h}$ and $48 \mathrm{~h}$ incubation times, and cell viability and damage were assessed. Positive controls were prepared exposing cells to increasing concentrations of STS and SDS. At each incubation time, cellular supernatants were transferred onto a 96-well plate for LDH assay, while cells were washed with DPBS and tested for their viability. Mitochondrial activity was evaluated by recording absorbance at $490 \mathrm{~nm}$ according to manufacturer's instructions, corresponding to the conversion of tetrazolium salt [3-(4,5-dimethylthiazol-2-yl)-5(3-carboxymethoxyphenyl)-2-(4-sulfophenyl)-2H] (MTS) into its reduced formazan form. The amount of ATP was detected by recording luminescent signal after treatment. Both absorbance and luminescence readings were recorded with the microplate reader Synergy4 (BioTek Instruments, Inc.), and results of three independent experiments reported as a ratio between absorbance and luminescence average values of CCL-treated cells and controls.

\section{Cellular interaction studies}

The interaction of CCL with cells was studied by fluorescence microscopy. Cells were treated from $30 \mathrm{~min}$ to $24 \mathrm{~h}$ either with CFloaded CCL or CF. After treatment, cellular supernatants were removed, cells were washed three times with PBS and fixed with $3.7 \%$ formaldehyde for $10 \mathrm{~min}$. After three washes with PBS, coverslips were mounted with $80 \%$ glycerol.

Fluorescence microscopy was carried out with a Leica DMI 6000B (Leica Microsystems, Wetzlar, Germany) equipped with Leica HCX PL APO $63 \times / 1.4$ objective lens. Illumination was provided via Leica EL6000 mercury metal halide lamp. CF was excited at $480 \mathrm{~nm}$ and fluorescence emission was recorded at $527 \mathrm{~nm}$ with Leica DFC360 FX CCD camera. Confocal laser scanning microscopy was carried out with Leica TCS SP5 II system utilizing an argon ion laser for excitation at $488 \mathrm{~nm}$. Sample fluorescence was recorded on a photomultiplier tube at wavelengths greater than $500 \mathrm{~nm}$. Objective lens used was HCX PL APO lambda blue $63 \times / 1.4$ with the pinhole set to $121 \mu \mathrm{m}$. All fluorescence images obtained from three independent experiments were acquired and analyzed with Leica LAS AF and ImageJ (Schneider et al., 2012) software.

\section{Statistical analysis}

Data analysis was performed by unpaired Student's T test with GraphPad Prism program (GraphPad Software, Inc., San Diego, USA; www.graphpad.com).

\section{Results and Discussion}

\section{General considerations}

To overcome limitations associated to many basic preparation techniques for liposomes, including the difficulty for scaling up to industrial production, an ethanol injection method based on controlled injection of organic solution was employed [32]. In general, ethanol injection and its modification involves the dissolution of lipids into ethanol (or alcohols as methanol or isopropanol) followed by rapid addition of organic solution into water.

Despite the easy protocol, further improvements to ethanol injection procedure are needed to improve the general pharmaceutical characteristics of produced vesicles, including size uniformity, batch to batch reproducibility and predictability. To this aim, in this current paper the ethanol injection protocol was performed by strictly controlling crucial experimental parameters, namely: (a) the rate of ethanol injection, (b) the stirring of chitosan-containing aqueous phase and (c) the geometry of impeller and glass reactor.

This procedure allowed a controlled ethanol diffusion in water, resulting in a precise and reproducible self-organization of lecithin and chitosan molecules and leading to homogenous CCL formulations.

\section{Preparation of CCL}

As previously stated, the main aim of current paper is the production of CCL by a controlled ethanol injection method resulting in high batch-to-batch reproducibility of CCL with narrow size distribution. To this purpose, a crucial step (i.e. the mixing of lecithin and chitosan solutions) was accomplished in a controlled manner by a syringe pump under mechanical stirring in a glass reactor. The effect of different lecithin to chitosan ratios were investigated, namely: 5, 10, 20, 50, 75 and 100. Hereafter, the resulting CCL are indicated as CCL-R5, CCL-R10, CCL-R20, CCL-R50, CCL-R75 and CCL-R100 throughout the text. Ethanol injection involves the dissolution of lipids into ethanol (or alcohols as methanol or isopropanol) followed by rapid addition of organic solution into water. The addition of lipids to the water phase, at temperatures above $\mathrm{T}_{\mathrm{m}}$, causes the self-assembling of lipids into liposomes, which are coated with chitosan molecules by electrostatic interactions. The main advantage of ethanol injection method resides in formation of liposomes with a quite narrow size distribution in one step, so avoiding degradation or oxidation of both lipids and drugs.

\section{Dimensional characterisation of CCL}

Dimensional and morphological characterisation of liposomes is required since their physico-chemical properties and biological behaviour are strongly related to their size, shape and lamellarity.

The European Commission defines a nanomaterial as a natural, incidental or manufactured material containing particles, in an unbound state, as an aggregate or as an agglomerate and where, for 50 $\%$ or more of the particles in the number size distribution, one or more external dimensions is in the size range 1-100 nm [33]. According to this definition, the size distribution of nanomaterials in a liquid phase plays an important role with respect to the final clinical application. 

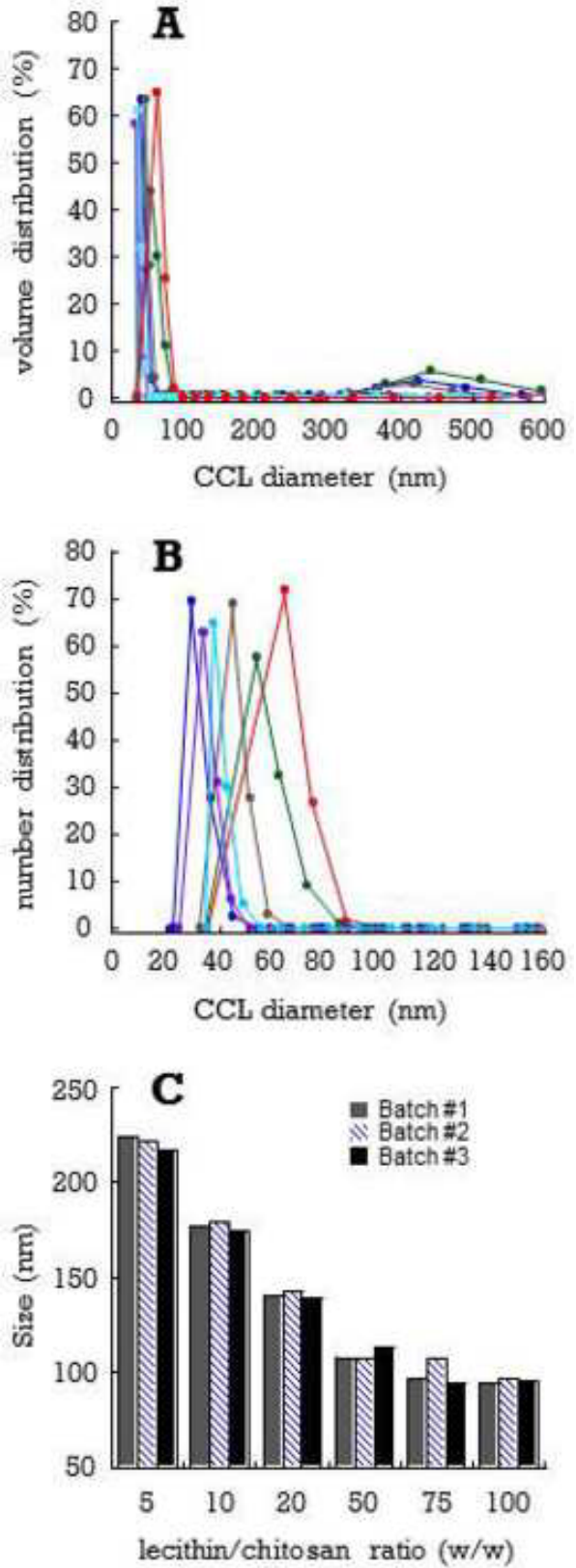

Figure 1: Effect of different lecithin/chitosan ratios on dimensional characteristics of CCL. Size distribution plot by volume (A) and by number (B) obtained with the Zetasizer 3000 PCS instrument. CCL were prepared at different lecithin/chitosan ratios (w/w), namely: 5 (red line), 10 (green line), 20 (blue line), 50 (grey line), 75 (purple line) and 100 (light blue line). Data of panel C refer to Z-average of three different CCL preparations.

Nanoparticles can indeed form secondary particles by soft or hard agglomeration mechanisms. To this end, dynamic light scattering (DLS) is widely used as an effective technique for determining the average nanoparticle size in suspensions and possible presence of nanoparticle assemblages. It is known that nanoparticle size distributions analysed by DLS strongly depend on instruments and analytical procedures used, so two different dynamic light scattering instruments were employed. All CCL samples were comparatively analysed by two DLS instruments respectively equipped with a detector positioned at $90^{\circ}$ and in backscattering mode.
The influence of different lecithin/chitosan ratios on CCL size was investigated, and DLS results are reported in Figure 1. An increase of lecithin to chitosan ratios from 5 to 100 , resulted in a progressive decrease of mean particle size (from $\sim 220$ to $\sim 100 \mathrm{~nm}$ ). At ratios equal to $10,20,50$ and 75 a small secondary peak was detectable only in volume size distribution (Figure 1A). Notably, batch-to-batch analysis of CCL sizes and size distributions revealed that the preparation procedure is highly reproducible (Figure 1C).

To confirm the relationship between dimensions and lecithin to chitosan ratios, CCL formulations were also analysed by a Zetasizer Nano ZS that utilizes a non-invasive backscattering arrangement (around 170 degrees) for increased sensitivity. In Figure 2 is reported the comparative size analysis of CCL-R5, CCL-R10 and CCL-R20 performed by the two instruments. Results show the same trend of Figure 1, with Z-average values generally 10-30 nm smaller in a backscattering instrument compared to the $90^{\circ}$ instrument.

\section{Morphological characterisation of CCL}

CCL morphology was analysed by TEM in order to characterise their architecture, lamellarity and size estimation. TEM images for CCL-R5, CCL-R10 and CCL-R20 are reported in Figure 3. All samples were predominantly constituted of multilamellar, spheroidal vesicles with diameters ranging from $\sim 20 \mathrm{~nm}$ to over $200 \mathrm{~nm}$. The lamellae thickness (shown in bright in negative stain TEM images) ranges between 2.3 and $3.5 \mathrm{~nm}$. Typical repetition distance between lamellae in close contact is 3.8 to $5 \mathrm{~nm}$. CCL-R 5 sample appeared the most heterogeneous by shape and size (Figure 3A). The majority of particles were indeed spheroidal, but elongated, straight, cylindrical vesicles, possibly cochleates were also frequently detectable in this sample. Cochleates are precipitates of cylindrical, elongated phospholipid aggregates that generally form upon stepwise addition of divalent inorganic cations (i.e. calcium ions) or polycations into small unilamellar vesicles prepared with phospholipid mixtures containing negatively charged amphiphiles, such as phosphatidylserine (or phosphatidylglycerol). Cochleate cylinders are rolled bilayers with a small interlamellar aqueous space due to cation interactions with negatively charged lipids. CCL-R10 (Figure 3B) and CCL-R20 (Figure 3C) samples did not show elongated particles but only spheroidal ones with majority of them having diameters smaller than $150 \mathrm{~nm}$. A major difference between CCL-R10 and CCL-R20 samples seems to be that the former has lamellae more tightly bound while the latter has more irregularly separated lamellae.

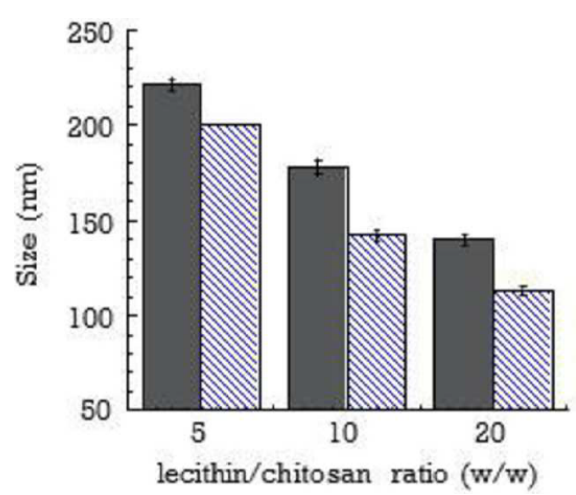

Figure 2: Comparative dimensional analysis of CCL. Dimensional characteristics expressed as Z-average are shown for Zetasizer 3000 (grey bars) and Zetasizer Nano ZS (striped bars) instrument. CCL were prepared with the indicated lecithin/chitosan ratios. Data are means of three independent batches \pm standard deviation. 


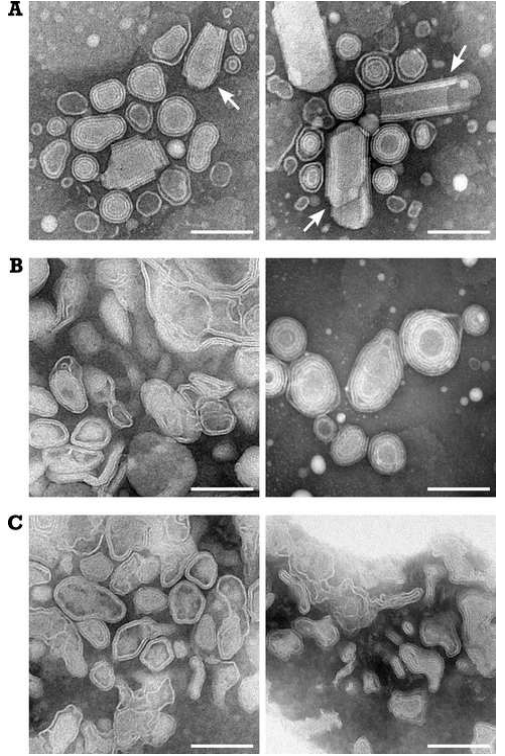

Figure 3: Negative-stain TEM images of CCL. Two representative images are shown for each sample: $C C L$ were prepared with different lecithin/chitosan ratios (w/w), namely: $5(A), 10(B)$ and $20(C)$. Arrows indicate the elongated, cylindrical structures. Scale bar indicates $100 \mathrm{~nm}$.
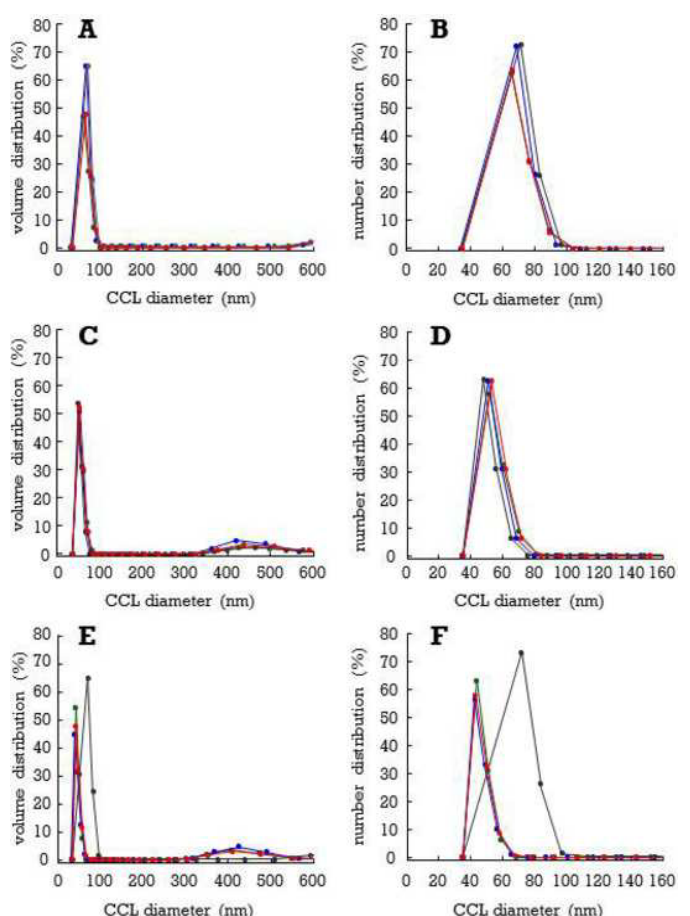

Figure 4: Long-term stability of CCL. Size distribution plot by volume (A, C, $E)$ and by number $(B, D, F)$ of $C C L$ prepared with different lecithin/chitosan ratios (w/w), namely: $5(A, B), 10(C, D)$ and $20(E, F)$. Measurement were performed immediately after preparation (red line) and after 15 (green line), 30 (blue line) and 60 days (grey line).

\section{Effect of aging on CCL}

One of the major hurdles in liposome use as drug delivery systems is represented by their long-term instability in suspensions. Generally, when liposomes encounter one another in suspension, they are prone to form aggregates and eventually fuse, resulting in an increase of liposome mean diameter and evident sediment formation. As pharmaceutical products, liposomes must be stable during the storage period and remain uniform in size up to the expiration date. Therefore, in the current study, a considerable attention was given to evaluate the stability of CCL suspensions over time. Figure 4 reports the stability, in term of size analysis, of CCL-R5, CCL-R10 and CCL-R20 stored in glass vials at $25^{\circ} \mathrm{C}$ up to 60 days. A good stability of CCL without agglomeration or sedimentation was observed for CCL-R5 (Figures 4A and $4 \mathrm{~B}$ ) and CCL-R10 (Figures 4C and 4D). Interestingly, CCL-R20 (Figure 4E and 4F) that contains a lowest amount of chitosan, showed a slight increase of particle size after two months of storage. Small amount of surface-adsorbed chitosan in CCL-R20 favours liposome vesicle interactions, while higher chitosan amounts (CCL-R5 and CCL-R10) render liposomes much more negatively charged and stable for long-time. Therefore, the strategy of liposome charging produces particle-stabilized liposomes that repel one another due to electrostatic repulsion forces.

\section{Effect of active molecule encapsulation}

It is well known that conventional liposomes possess the ability to encapsulate small molecules in the core, in the bilayer or on their surface. The hydrophilic dye molecule, carboxyfluorescein, was encapsulated in CCL for testing the effect of an encapsulated drug on dimensional and zeta-potential properties of CCL. Moreover, CF made the internal volume fluorescent, facilitating liposome visualization under fluorescence microscopy. To this aim, CF loaded CCL were prepared by adding CF to the ethanolic solution in which lecithin was solubilized. CF loading yield was determined after gel filtration chromatography that was accomplished in order to separate CF loaded CCL from free CF. CF entrapment for all lecithin/chitosan ratios was over $80 \%$

With respect to dimensional effect of CF on CCL (Figure 5), no significant differences in size were observed comparing empty and CF loaded CCL, for all tested lecithin/chitosan ratios (i.e. CCL-R5, CCL-R10 and CCL-R20). As previously noted for empty CCL, CF loaded CCL nanoparticle progressively decreased in size as lecithin/ chitosan ratio increases (Figure 6A).

Zeta-potential analysis confirmed that CF encapsulation had limited effect on electrokinetic potential of CCL. As expected, CCL were positively charged as a result of chitosan interaction with liposome surface starting at $+45 \mathrm{mV}$ for CCL-R5 sample. Decreasing chitosan amounts, displayed a concomitant decrease of zeta potential in CCL-R10 and CCL-R20, of +43 and $+34 \mathrm{mV}$, respectively (Figure $6 \mathrm{~B})$. At all ratios, CF loaded CCL showed a less positive zeta potential due to negative charges of CF. The limited decrease was attributed to the relatively low concentration of $\mathrm{CF}$ in coated liposomes.

\section{Cytotoxicity and cellular interaction studies}

To evaluate CCL cytotoxicity, we used A549 and Caco- 2 cell lines as in vitro models respectively of human lung and intestinal epithelia. Before investigating cytotoxicity, DLS measurements on CCL in cell culture media at the various incubation times were performed to evaluate liposome integrity and stability during experiments. DLS data of CCL showed a similar pattern as CCL in 10\% ethanolic suspension (data not shown). Cell viability and damage were determined after $2 \mathrm{~h}$, $6 \mathrm{~h}, 24 \mathrm{~h}$ and $48 \mathrm{~h}$ exposure to increasing CCL concentrations (Figure 7 and 8 and supplemental Figure 1 and 2 ). Mitochondrial activity impairment was observed only on A549 cells exposed to CCL for 48 

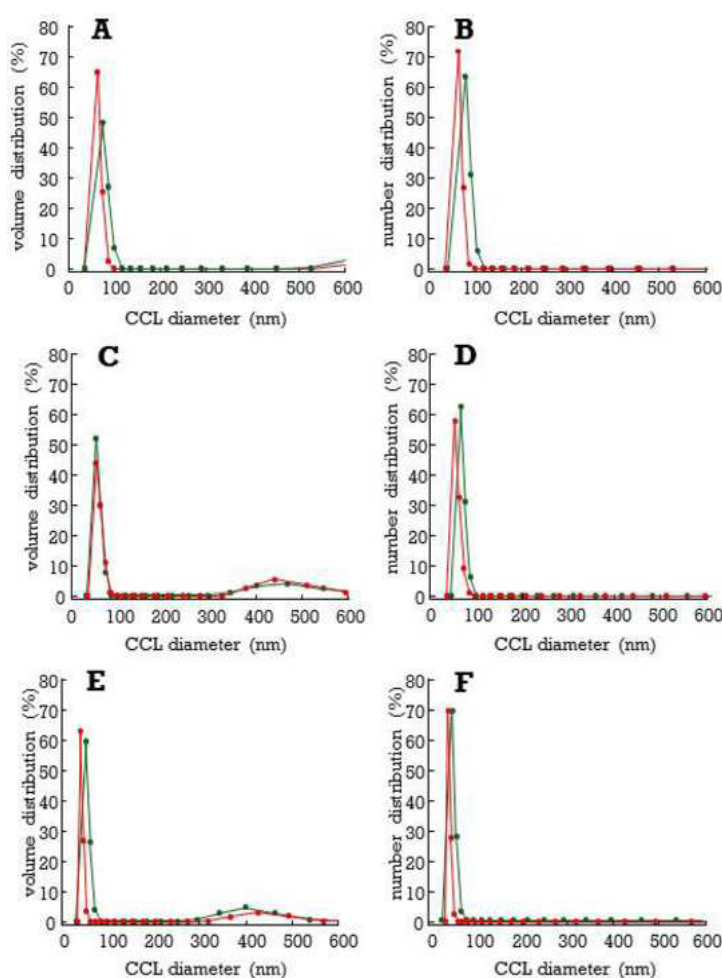

Figure 5: Size distribution comparison between empty and CF loaded CCL Size distribution plot by volume (A, C, E) and by number (B, D, F) of empty (red line) and CF loaded CCL (green line) prepared with different lecithin/chitosan ratios $(w / w)$, namely: $5(A, B), 10(C, D)$ and $20(E, F)$.

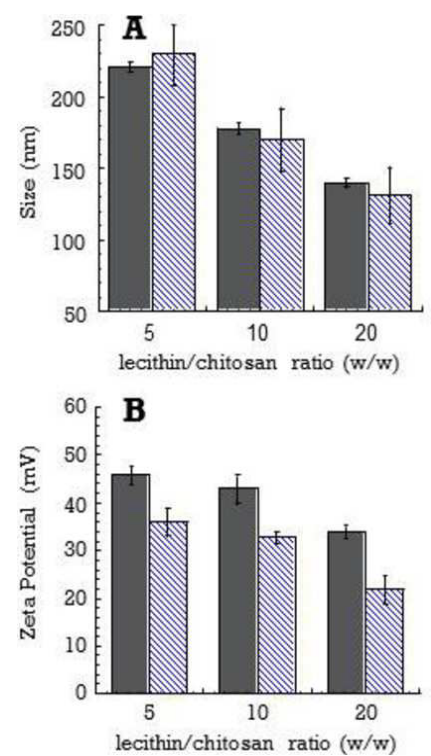

Figure 6: Size distribution and zeta potential comparison between empty and CF loaded CCL. Comparative analysis of dimensional $(A)$ and zeta-potential characteristics (B) of empty (grey bars) and CF loaded CCL (striped bars). Samples were prepared with indicated lecithin/chitosan ratios. Data are means of three independent batches \pm standard deviation.

h (Figure 7 and supplemental Figure 1). In particular, CCL-R5 showed significant reduction from $114 \mu \mathrm{g} / \mathrm{mL}$ (expressed as concentration of

lecithin), while CCL-R10 and CCL-R20 affected cell viability from $456 \mu \mathrm{g} / \mathrm{mL}$. These results were confirmed by ATP content reduction (supplemental Figure 3). No effects on Caco-2 cell viability were observed at all incubation times, as shown in Figure 8 and supplemental Figure 2 and 4 . No LDH release into cell culture media was observed in both cell lines, suggesting that CCL did not affect membrane integrity.

CCL interaction with both cell lines was investigated by using wide-field and confocal fluorescence microscopy. In particular, cells were treated with CF labeled CCL at different incubation periods. At short time exposure (30 $\mathrm{min})$, CF labelled CCL were already found to bind both A549 and Caco-2 cells (Figure 9 and supplemental Figure 5 for higher image resolution and dynamic range). In A549 samples, cell associated fluorescence is better spread than Caco-2, with higher signal at 30min for CCL-R5 and lower for CCL-R20. All samples showed a similar trend in reduction of unbound particle fluorescence at 6 and 24 h (Figures 10 and 11 and supplemental Figures 6 and 7). However, even if Caco-2 samples generally exhibited less fluorescence intensity, at 6 and $24 \mathrm{~h}$ the fluorescence signal is well localized on cells. Labelled CCL interaction was compared with $\mathrm{CF}$ alone, which was not found nor as unbound particle neither on cell membranes, at all incubation times. The overall analysis of samples fluorescence revealed that total amount of fluorescent particles was higher for CCL-R5 and decreased with the

$\mathbf{A}$

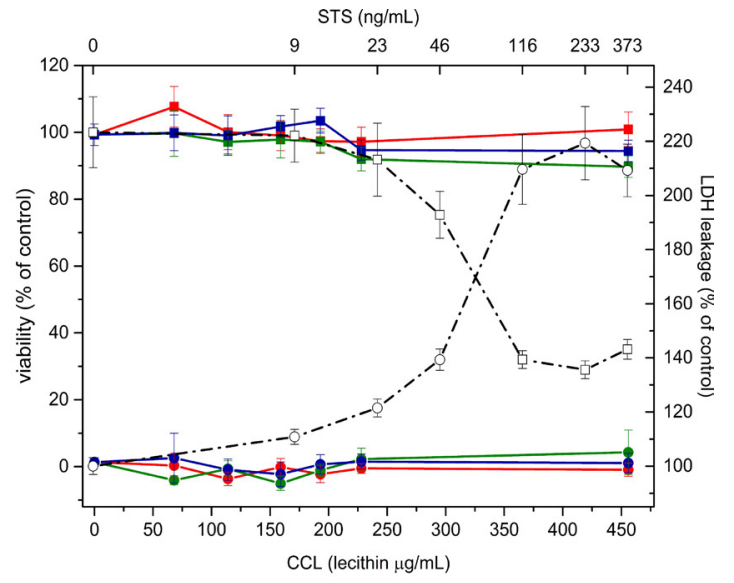

$\mathrm{STS}(\mathrm{ng} / \mathrm{mL})$

B

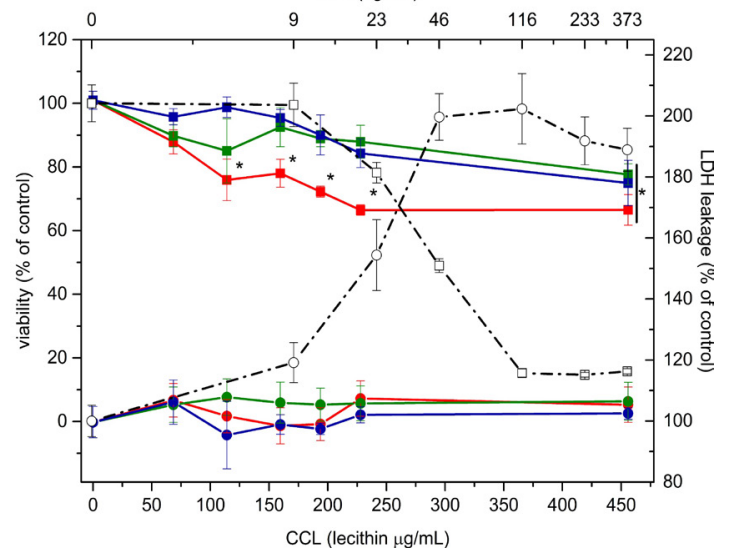

Figure 7: Cytotoxicity on A549 cells. Viability (squares) and membrane integrity (circles) assays of A549 cells exposed to increasing CCL concentrations (CCL-R5 red line, CCL-R10 green line, CCL-R20 blue line) for $24 \mathrm{~h}(\mathrm{~A})$ and $48 \mathrm{~h}(\mathrm{~B})$. Positive control curves obtained with STS (dash-dot black lines) are reported. $\left({ }^{*} \mathrm{P}<0.05\right)$. Results are shown as mean \pm standard deviation of 3 independent experiments. 
Citation: Venturini M, Mazzitelli S, Mičetić I, Benini C, Fabbri J, et al. (2014) Analysis of Operating Conditions Influencing the Morphology and In vitro Behaviour of Chitosan Coated Liposomes. J Nanomed Nanotechnol 5: 211. doi: 10.4172/2157-7439.1000211

Page 7 of 8

$\boldsymbol{A}$

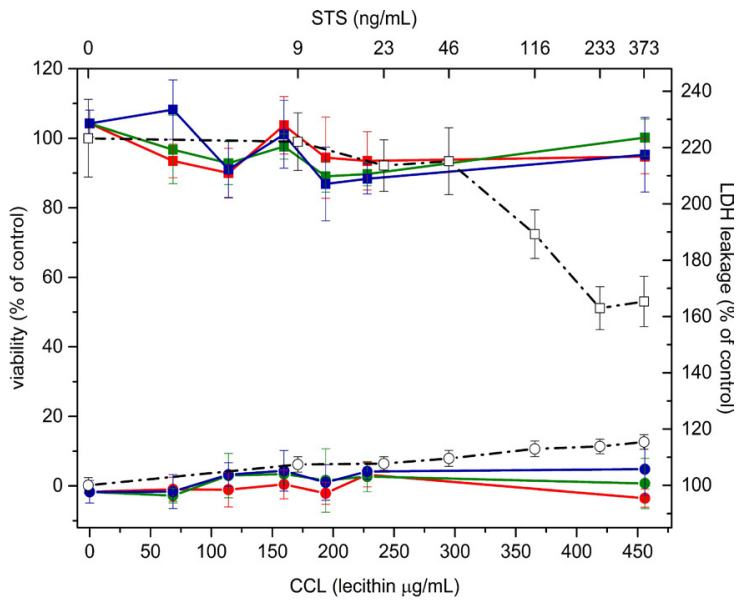

B

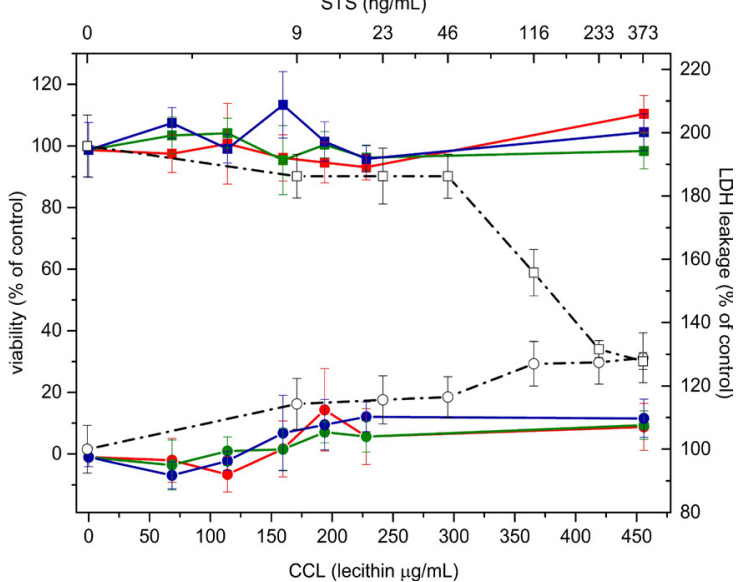

Figure 8: Cytotoxicity on Caco-2 cells. Viability (squares) and membrane integrity (circles) assays of Caco-2 cells exposed to increasing CCL (CCL-R5 red line, CCL-R10 green line, CCL-R20 blue line) concentrations for $24 \mathrm{~h}$ (A) and $48 \mathrm{~h}$ (B). Positive control curves obtained with STS (dash-dot black lines) are reported. $\left({ }^{*} P<0.05\right)$. Results are shown as mean \pm standard deviation of 3 independent experiments.

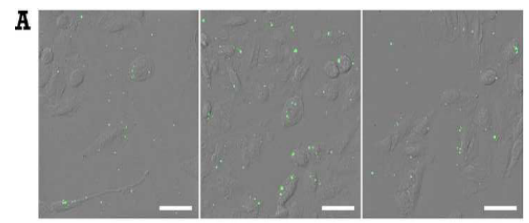

B

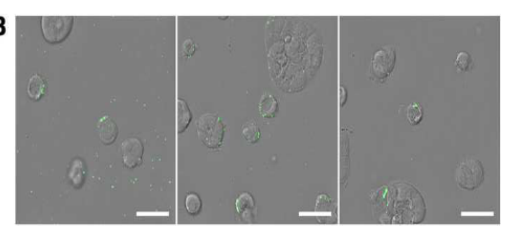

Figure 9: CCL interactions with A549 and Caco-2 cells. Representative confocal laser scanning microscopy images of CF loaded CCL after 30 minutes of incubation. A549 cells (A) and Caco-2 cells (B) exposed to CCL with lecithin/ chitosan ratios of 5 (left), 10 (center) and 20 (right). Images are the overlay of fluorescence and transmitted light (DIC) signals. Scale bar indicates $50 \mu \mathrm{m}$.

increase of lecithin/chitosan ratio. Finally, morphology of both cell types was not affected by the treatments confirming non-toxic effects of CCL at observed time points.

\section{Conclusions}

Coated liposomes composed of lecithin (primarily constituted of phosphatidylcholine) and high molecular weight chitosan represent an interesting carrier for nanomedicine applications. The structure of nanoparticles is formed by ionic interactions between lecithin and chitosan providing a prominent surface charge resulting in bioadhesive properties. CCL sizes prepared at different lecithin to chitosan weight ratio was always below $200 \mathrm{~nm}$ and TEM analysis revealed that nanoparticles were mainly characterized by a spherical shape with the presence of some cochleates at low lecithin to chitosan ratios. Furthermore, liposome coating by a chitosan layer was confirmed by TEM images and zeta potential analysis. In addition, our results demonstrated that surface coating with chitosan preserved liposome stability at least for 60 days. CF has been successfully encapsulated in CCL allowing to investigate CCL interactions with cells by fluorescence microscopy. Presented data indicate that the use of appropriate lecithin
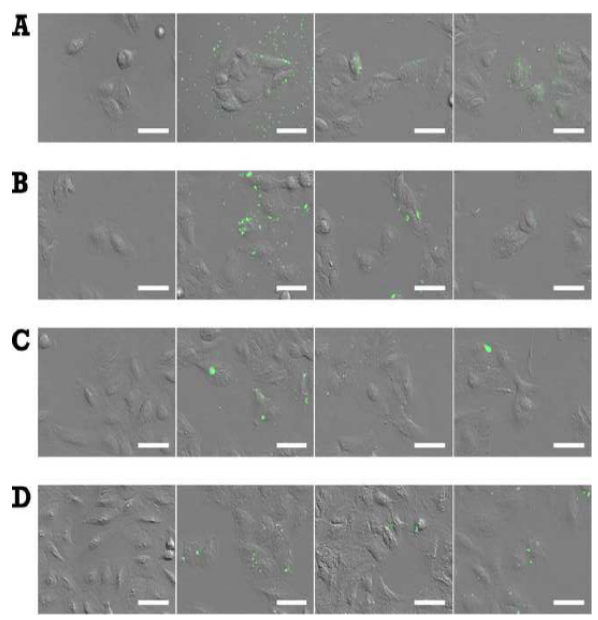

Figure 10: CCL interactions with A549 cells at increasing incubation times. Representative fluorescence images of A549 cells with CF loaded CCL at increasing incubation time. Incubation times of $30 \mathrm{~min}(\mathrm{~A}), 2 \mathrm{~h}(\mathrm{~B}), 6 \mathrm{~h}(\mathrm{C})$ and $24 \mathrm{~h}(\mathrm{D})$ are shown. Rows show from left to right untreated cells (left) and cells treated with CCL-R5, CCL-R10 and CCL-R20. Fluorescence signals are overlaid on transmitted light (DIC) images. Scale bar indicates $50 \mu \mathrm{m}$.

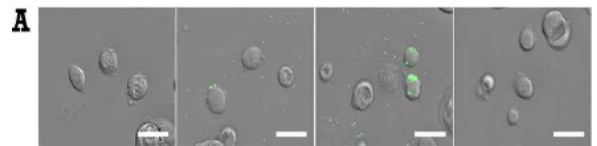

B
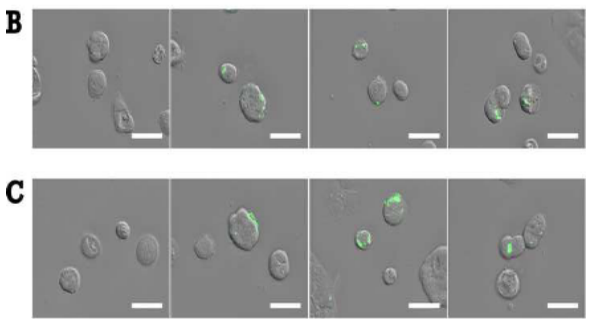

Figure 11: CCL interactions with Caco-2 cells at increasing incubation times. Representative fluorescence images of Caco-2 cells with CF loaded CCLs at increasing incubation time. Incubation times of 30 min (A), $6 \mathrm{~h} \mathrm{(B)}$ and $24 \mathrm{~h}(\mathrm{C})$ are shown. Rows show from left to right untreated cells (left) and cells treated with CCL-R5, CCL-R10 and CCL-R20. Fluorescence signals are overlaid on transmitted light (DIC) images. Scale bar indicates $50 \mu \mathrm{m}$. 
Citation: Venturini M, Mazzitelli S, Mičetić I, Benini C, Fabbri J, et al. (2014) Analysis of Operating Conditions Influencing the Morphology and In vitro Behaviour of Chitosan Coated Liposomes. J Nanomed Nanotechnol 5: 211. doi: 10.4172/2157-7439.1000211

to chitosan ratios results in a formation of non-toxic, highly stable formulations optimally suitable as drug delivery systems.

\section{References}

1. Misra A, Ganesh S, Shahiwala A, Shah SP (2003) Drug delivery to the centra nervous system: a review. J Pharm Pharm Sci 6: 252-273.

2. Zhang L, Pornpattananangku D, Hu CM, Huang CM (2010) Development of nanoparticles for antimicrobial drug delivery. Curr Med Chem 17: 585-594.

3. Capretto L, Mazzitelli S, Colombo G, Piva R, Penolazzi L, et al. (2013) Production of polymeric micelles by microfluidic technology for combined drug delivery: Application to osteogenic differentiation of human periodontal ligament mesenchymal stem cells (hPDLSCs). Int J Pharmaceutics 440: 195-206.

4. Nastruzzi C, Cortesi R, Esposito E, Gambari R, Borgatti M, et al. (2000) Liposomes as carriers for DNA-PNA hybrids. J Control Release 68: 237-249.

5. Xu W, Ling P, Zhang T (2013) Polymeric micelles, a promising drug delivery system to enhance bioavailability of poorly water-soluble drugs. J Drug Deliv 2013: 340315

6. Akter N, Radiman S, Mohamed F, Reza MI (2013) Self-assembled potential bio nanocarriers for drug delivery. Mini Rev Med Chem 13: 1327-1339.

7. Nicolas J, Mura S, Brambilla D, Mackiewicz N, Couvreur P (2013) Design, functionalization strategies and biomedical applications of targeted biodegradable/biocompatible polymer-based nanocarriers for drug delivery. Chem Soc Rev 42: 1147-1235.

8. Alvarez-Román R, Naik A, Kalia YN, Guy RH, Fessi H (2004) Enhancement of topical delivery from biodegradable nanoparticles. Pharm Res 21: 1818-1825.

9. Barbosa-Barros L, Barba C, Rodríguez G, Cócera M, Coderch L, et al. (2009) Lipid nanostructures: self-assembly and effect on skin properties. Mol Pharm 6: 1237-1245.

10. Baroli B, Ennas MG, Loffredo F, Isola M, Pinna R, et al. (2007) Penetration of metallic nanoparticles in human full-thickness skin. J Invest Dermatol 127: 1701-1712.

11. Yanasarn N, Sloat BR, Cui Z (2009) Nanoparticles engineered from lecithinin-water emulsions as a potential delivery system for docetaxel. Int J Pharm 379: 174-180.

12. Alonso MJ, Sánchez A (2003) The potential of chitosan in ocular drug delivery. J Pharm Pharmacol 55: 1451-1463.

13. Scholfield CR (1981) Composition of soybean lecithin. J American Oil Chemists Soc 58: 889-892.

14. Schubert MA, Müller-Goymann CC (2005) Characterisation of surface-modified solid lipid nanoparticles (SLN): influence of lecithin and nonionic emulsifier. Eur $\mathrm{J}$ Pharm Biopharm 61: 77-86.

15. Felt O, Buri P, Gurny R (1998) Chitosan: a unique polysaccharide for drug delivery. Drug Dev Ind Pharm 24: 979-993.

16. Kumar MN, Muzzarelli RA, Muzzarelli C, Sashiwa H, Domb AJ (2004) Chitosan chemistry and pharmaceutical perspectives. Chem Rev 104: 6017-6084

17. Rodrigues S, Dionísio M2, López CR3, Grenha A4 (2012) Biocompatibility of chitosan carriers with application in drug delivery. J Funct Biomater 3: 615-641.

18. Chopra S, Mahdi S, Kaur J, lqbal Z, Talegaonkar S, et al. (2006) Advances and potential applications of chitosan derivatives as mucoadhesive biomaterials in modern drug delivery. J Pharm Pharmacol 58: 1021-1032.
19. Singh D, Ray AR (2000) A Biomedical applications of chitin, chitosan and their derivatives. Rev Macromol Chem Phys C40: 69-83.

20. Park JH, Saravanakumar G, Kim K, Kwon IC (2010) Targeted delivery of low molecular drugs using chitosan and its derivatives. Adv Drug Deliv Rev 62 : 28-41.

21. Dudhani AR, Kosaraju S (2010) Bioadhesive chitosan nanoparticles: Preparation and characterization. Carbohydrate Pol 81: 243-251.

22. Chadha R, Gupta S, Pathak N (2012) Artesunate-loaded chitosan/lecithin nanoparticles: preparation, characterization, and in vivo studies. Drug Dev Ind Pharm 38: 1538-1546.

23. Fernández-Urrusuno $R$, Calvo $P$, Remuñán-López $C$, Vila-Jato $\mathrm{JL}$, Alonso $M$ (1999) Enhancement of nasal absorption of insulin using chitosan nanoparticles. Pharm Res 16: 1576-1581.

24. Prego C, Torres D, Alonso MJ (2005) The potential of chitosan for the oral administration of peptides. Expert Opin Drug Deliv 2: 843-854.

25. Andrade F, Antunes F, Nascimento AV, da Silva SB, das Neves J, et al. (2011) Chitosan formulations as carriers for therapeutic proteins. Curr Drug Discov Technol 8: 157-172.

26. Ma Z, Lim TM, Lim LY (2005) Pharmacological activity of peroral chitosaninsulin nanoparticles in diabetic rats. Int J Pharm 293: 271-280.

27. Liu X, MA L, Mao Z, Gao C (2011) Chitosan-based biomaterials for tissue repair and regeneration. Adv Polym Sci 244: 81-128.

28. Sezer AD, Cevher E (2012) Topical drug delivery using chitosan nano- and microparticles. Expert Opin Drug Deliv 9: 1129-1146.

29. Phetdee M, Polnok A, Viyoch J (2008) Development of chitosan-coated liposomes for sustained delivery of tamarind fruit pulp's extract to the skin. Int J Cosmet Sci 30: 285-295.

30. Li N, Zhuang C, Wang M, Sun X, Nie S et al. (2009) Melatonin-loaded lecithin chitosan nanoparticles: Physicochemical characterisation and permeability through Caco-2 cell monolayers. Int J Pharmaceutics 379: 131-138.

31. Gonçalves MC, Mertins O, Pohlmann AR, Silveira NP, Guterres SS (2012) Chitosan coated liposomes as an innovative nanocarrier for drugs. J Biomed Nanotechnol 8: 240-250.

32. Stano P, Bufali S, Pisano C, Bucci F, Barbarino M, et al. (2004) Nove camptothecin analogue (gimatecan)-containing liposomes prepared by the ethanol injection method. J Liposome Res 14: 87-109.

33. Kato H, Nakamura A, Takahashi K, Kinugasa S (2012) Accurate size and sizedistribution determination of polystyrene latex nanoparticles in aqueous medium using dynamic light scattering and asymmetrical flow field flow fractionation with multi-angle light scattering. Nanomaterials 2: 15-30. 